\title{
Strates
}

STRATES Matériaux pour la recherche en sciences sociales

5 | 1990

Conjuguer stratégies et territoires?

\section{Géostratégies du capital et de l'État à Taïwan et Singapour}

\section{Catherine Paix et Michèle Petit}

\section{(2) OpenEdition \\ 1 Journals}

Édition électronique

URL : http://journals.openedition.org/strates/1372

DOI : $10.4000 /$ strates. 1372

ISSN : $1777-5442$

Éditeur

Laboratoire Ladyss

Édition imprimée

Date de publication : 31 décembre 1990

ISSN : 0768-8067

Référence électronique

Catherine Paix et Michèle Petit, "Géostratégies du capital et de l'État à Taïwan et Singapour », Strates [En ligne], 5 | 1990, mis en ligne le 16 mars 2007, consulté le 08 septembre 2020. URL : http:// journals.openedition.org/strates/1372 ; DOI : https://doi.org/10.4000/strates.1372

Ce document a été généré automatiquement le 8 septembre 2020

Tous droits réservés 


\title{
Géostratégies du capital et de l'État à Taïwan et Singapour
}

\author{
Catherine Paix et Michèle Petit
}

\begin{abstract}
«Dans la stratégie, le facteur déterminant est le moment, le facteur accessoire, les plans. »

Guanzi ${ }^{1}$

«Le moment est à la fois la configuration générale d'une époque - la situation historique - et cet instant unique et privilégié, cette occasion précise qui ne reviendra pas et ouvre par là même des perspectives d'élévation ou de profit à l'individu entreprenant et audacieux qui sait saisir sa chance en se laissant porter par la tendance, la conjoncture ».

Jean Lévi, Les fonctionnaires divins ${ }^{2}$.
\end{abstract}

1 Enjeux géopolitiques majeurs en Asie pour les pays capitalistes centraux, les États de Taïwan et Singapour ont été soumis dès leur création à des ingérences extérieures qui ont pesé très lourdement sur l'orientation des politiques gouvernementales et la restructuration de leur système économique et social à l'issue de la période coloniale. Devenus des lieux privilégiés de délocalisation des firmes nord-américaines et nippones - et plus largement du capital transnational - leur croissance interne s'est appuyée, et repose toujours, sur une extraversion et une dépendance très importantes de leur économie vis-à-vis des capitaux et marchés extérieurs.

2 Il n'en demeure pas moins qu'à l'intérieur d'un champ de possibles délimité par les contraintes économiques, sociales et politiques auxquelles ils se sont trouvés confrontés à différentes échelles, États et entrepreneurs locaux ont développé et affirmé sur le long terme des stratégies d'intégration à l'économie et à l'espace mondial qui ont été décisives dans le développement de ces pays et la position « d'exception » qu'ils occupent actuellement parmi les pays du Tiers Monde.

Il revient en effet aux gouvernements d'avoir joué des rapports de domination/ dépendance dans lesquels se sont trouvés placés ces pays, en les retournant dans le sens de l'accumulation interne et d'une diffusion des effets de la croissance dans l'ensemble de leur territoire national, et en créant sur ces bases les conditions simultanées d'une attraction soutenue des capitaux étrangers et d'une progression continue des propres capacités d'affirmation de ces économies. Il revient aussi aux 
entrepreneurs locaux d'avoir positivé l'ensemble des atouts et des réseaux de relations dont ils pouvaient disposer aux échelles nationale et internationale pour s'associer aux firmes étrangères, et d'avoir simultanément mis à profit cette association pour consolider leurs capacités économiques et créer les conditions d'une adaptation constante de leurs firmes aux mutations de l'économie mondiale.

4 Les politiques et les pratiques entrepreneuriales qui matérialisent ces stratégies se sont conjuguées, il est vrai, avec les potentialités économiques et humaines de ces espaces nationaux et avec les possibilités qu'offrait leur position clé dans l'échiquier international :

5 - à Taïwan, un potentiel industriel non négligeable et diversifié dans l'industrie lourde ; une agriculture fondée sur la petite exploitation familiale, dont la rentabilité avait été considérablement élevée sous la colonisation japonaise ${ }^{3}$; une main-d'œuvre et une élite formées à l'industrie ; une répartition équilibrée des infrastructures et une tradition de dispersion de la petite industrie en milieu rural, offrant la possibilité de développer les petites entreprises dans l'ensemble du territoire national; une aide matérielle et financière considérable des États-Unis, pour redresser l'économie après la guerre ; et pour les entrepreneurs, des relations privilégiées avec le Japon.

6 - à Singapour, une position géostratégique essentielle pour les États-Unis au moment de la guerre du Vietnam, qui a coïncidé avec l'amorce d'une délocalisation des firmes américaines; une situation d'hégémonie portuaire à l'échelle régionale, et l'existence d'une bourgeoisie forte de ses fonctions d'intermédiaire entre les firmes étrangères et les pays environnants durant la période coloniale.

7 Dans les deux cas, les politiques gouvernementales ont également correspondu très étroitement aux objectifs des États-Unis, qui ont marqué leur volonté de créer dans ces pays les conditions d'une stabilité politique durable par la voie de la croissance économique, pour s'en faire des points d'appui privilégiés en Asie, et qui sont intervenus directement dans ce sens auprès des États. Quant à la consolidation d'un capital local, elle n'est pas sans liens avec la stratégie des firmes étrangères qui ont recherché l'association avec des intermédiaires locaux.

8 Les logiques d'affirmation économique qui informent les pratiques économiques et politiques de ces acteurs, et les principes d'équilibre, de simultanéité d'action, d'interaction d'échelles qui en sont constitutifs ne sont pas pour autant réductibles à une simple adaptation des acteurs aux structures et aux contraintes économiques et politiques, ou à une rationalité économique indépendante de l'histoire de leurs auteurs.

9 Dans les deux cas, les rapports que ceux-ci ont instaurés entre leur espace interne et l'extérieur relèvent de finalités et de choix propres qu'ils ont définis au moment de la constitution de ces États, qu'ils ont reconduits sur le long terme, et qui ont pesé durablement sur le développement de ces pays.

10 Il nous a semblé qu'on ne pouvait comprendre la spécificité de leurs stratégies qu'en analysant les circonstances précises qui ont présidé à leur élaboration : qu'il fallait alors identifier la position économique, sociale, politique et territoriale de ces acteurs à ce moment là, les objectifs et représentations territoriales qu'ils avaient développés, dans le champ des possibles qui leur était alors ouvert aux échelles nationale et internationale, et la façon dont ils avaient actualisé leur mémoire dans ce contexte.

11 C'est à montrer comment ces acteurs se sont "emparés du temps et de l'espace $~^{4}$ dans l'élaboration de leurs stratégies que nous nous sommes par conséquent attachées. La 
compréhension de la dynamique propre de ces espaces nationaux, mais aussi ce qui définit une stratégie, et le type d'analyse que son identification implique, ne peuvent en être, à notre avis, que clarifiés.

Une forte ambition territoriale

Tant à Taïwan qu'à Singapour, les stratégies de développement et d'intégration à l'extérieur adoptées par les classes dirigeantes sont tout à fait indissociables de la situation de double rupture territoriale et politique dans laquelle elles se sont trouvées placées lorsqu'elles ont accédé au pouvoir : rupture avec les espaces nationaux dont ces pays étaient jusque-là partie intégrante (la Chine et la Malaisie), doublée d'une interruption des relations économiques avec les espaces auxquels les liait le système colonial (l'Indonésie et le Japon), mais aussi rupture par rapport à la société, à laquelle ces gouvernements ont imposé leur autorité par des actes d'opposition violente dans des situations de crise économique et sociale aiguë: autant de situations qui ont impliqué un renversement complet de perspectives, tant au niveau du modèle de développement à adopter que du rattachement de ces pays à l'espace mondial.

À Taïwan, le gouvernement nationaliste, qui s'était imposé de l'extérieur après la victoire des communistes en Chine en 1949, était privé de tout ancrage dans la société taïwanaise qui lui était en grande partie opposée, du fait notamment des malversations de l'armée nationaliste après la guerre et des massacres qu'elle y avait perpétrés en 1947 ; il avait à faire face à une crise économique sans précédents que seule une restructuration radicale de l'économie pouvait résorber et ses interventions à ce niveau étaient un enjeu d'autant plus crucial pour le régime que celui- ci était sous le coup de ses échecs en Chine.

14 À Singapour, où le gouvernement s'était coupé de la population dès les premières années qui avaient suivi son accès au pouvoir en 1959, la classe dirigeante n'était pas à l'abri d'une résurgence des mouvements révolutionnaires. Alors que le retrait de la Fédération de Malaisie et la montée du nationalisme dans les pays voisins remettaient en cause les possibilités d'un développement de l'industrie de substitution tournée vers le marché malais et menaçaient l'économie d'entrepôt, le gouvernement avait à faire face à un taux de chômage très élevé et à une forte dégradation des conditions de vie de la population. Il était au défi de prouver ses capacités à restaurer l'économie, dans les limites d'un territoire très restreint.

Dans les deux cas, les classes dirigeantes étaient donc dans une position de très grande fragilité, qui leur imposait de se trouver de nouveaux partenaires extérieurs, mais aussi de réorienter l'économie et d'instaurer la stabilité politique, en dotant leur régime de la base sociale qui leur manquait pour se maintenir au pouvoir. Mais ce qu'il est important de souligner, c'est le fait que dans ces situations de double rupture, qui ont coïncidé avec les possibilités qu'offrait l'entrée dans l'orbite américaine, les gouvernements ont développé l'idée de prouver leur supériorité par rapport aux régimes des pays dont ils étaient exclus, en plaçant leur territoire en position d'« exception ", tant au niveau économique et social interne, qu'au niveau de sa place dans l'espace international.

À Singapour, avec l'éviction de la Malaisie et l'opportunité d'amorcer un processus d'industrialisation pour l'exportation en ouvrant l'économie aux firmes multinationales, les dirigeants sont rapidement passés d'une vision "régionaliste » et " nationale » du développement, à la représentation d'une ville " mondiale », « totale ». Et ils ont développé la volonté politique de préserver à la ville une position d'avantage 
comparé au sein de l'espace régional et international, en créant un écart de développement croissant avec les pays environnants.

À Taïwan, la position d'exil du gouvernement, dont l'objectif était de reprendre le pouvoir en Chine - pouvoir qu'il a toujours prétendu incarner -, et le soutien apporté par les États-Unis pour restaurer l'économie, ont renforcé chez les dirigeants la volonté de défier l'idéologie communiste, en prouvant les capacités du régime nationaliste à engager un processus de développement économique sur une base capitaliste, et en prouvant ses capacités à résorber les inégalités qui avaient été à l'origine de sa chute en Chine.

Dans des espaces "placés au-dessus d'eux-mêmes", comme Braudel le disait de la Méditerranée, par l'ampleur des enjeux géopolitiques qui s'y jouaient, les gouvernements de ces pays ont donc développé des ambitions sans commune mesure avec la dimension de leurs territoires et la position de grande vulnérabilité dans laquelle ils étaient - ambitions qui excédaient très largement la seule finalité politique de trouver une solution aux crises sociales et économiques auxquelles ils étaient confrontés.

19 À Singapour, ces ambitions ont été en phase, jusqu'à un certain point, avec celles de la bourgeoisie commerçante, qui avait fondé son expansion sur les échanges avec les pays voisins et qui entendait préserver son rôle hégémonique dans les relations régionales. À Taïwan plus encore, les ambitions gouvernementales ont été confortées par la couche potentielle des entrepreneurs sur laquelle a reposé en grande partie le développement industriel du pays : en position d'exclusion politique et de forte incertitude, celle-ci a cherché à s'affirmer par la voie de la « réussite » de ses entreprises pour s'imposer dans la société et vis-à-vis du pouvoir : c'est le cas de l'élite taïwanaise, qui était exclue de l'administration et du pouvoir politique - au mains de la bureaucratie nationaliste formée de Chinois du continent - et qui s'est tournée vers l'industrie pour retrouver une position sociale perdue ; c'est le cas aussi des industriels chinois du continent, qui pour certains partageaient les objectifs du gouvernement nationaliste, et qui ont voulu prouver leurs aptitudes entrepreneuriales pour se faire reconnaître dans la société taïwanaise et vis-à-vis de l'extérieur.

Pour les gouvernements, prouver leur supériorité en créant les conditions d'un développement économique à long terme et d'une stabilité politique durable, pour les entrepreneurs, s'imposer économiquement dans une certaine autonomie par rapport au pouvoir politique, tels ont donc été les enjeux majeurs : ces objectifs ont été des ressorts essentiels de leurs stratégies de développement et d'intégration à l'extérieur, sans lesquels on ne pourrait comprendre qu'ils aient cherché à tirer partie, comme ils l'ont fait, des opportunités internes et externes qui s'offraient à eux.

Une certaine façon de « s'emparer » du temps et de l'espace

Indissociables de leur position initiale et des objectifs qu'ils se sont alors fixés, les stratégies des gouvernements et des entrepreneurs de Taïwan et de Singapour le sont tout autant de la façon dont ils ont alors actualisé et réinterprété certains éléments de leur histoire, et plus largement de leur culture.

Dans les deux cas, les classes dirigeantes ont puisé de façon implicite ou explicite les grandes lignes de leurs stratégies dans l'idéologie républicaine chinoise, telle qu'elle avait été formulée par Sun Yat-sen - idéologie dont le gouvernement nationaliste de 
Taïwan était le garant, et qui avait beaucoup influencé les milieux de la diaspora chinoise de Singapour.

Sun Yat-sen avait rompu avec l'idéal d'autosuffisance qui avait longtemps marqué le monde chinois, et pensé le développement de la Chine dans un cadre planétaire, posant l'adaptation à l'économie internationale et l'assimilation des progrès de l'occident au cœur de sa problématique ${ }^{5}$; mais il avait envisagé l'intégration au système capitaliste et l'ouverture aux échanges internationaux sur la base d'avantages mutuels entre les nations, et avec l'idée de placer l'État en position de préserver l'intérêt national par rapport aux intérêts capitalistes nationaux et étrangers et d'assurer le développement équilibré des différents secteurs de l'économie, par le biais d'une planification et d'une étatisation partielle.

Ces grands principes, qui pouvaient s'accorder en de nombreux points avec les fortes capacités de contrôle territorial et d'intervention économique dont disposaient initialement les dirigeants de Taïwan et de Singapour, et avec les opportunités ouvertes au plan international, ont été largement repris par les gouvernements des deux pays. L'ouverture de l'économie au marché international et aux firmes et capitaux étrangers a été fortement encouragée, quand elle n'a pas été d'emblée recherchée. Mais, dans le cadre d'une forte intervention de l'État et de politiques très dirigistes à l'égard du secteur privé, cette ouverture n'a jamais été dissociée d'une valorisation d'ensemble des ressources économiques et humaines. Elle a aussi été accompagnée de mesures assurant simultanément le transfert des compétences étrangères à l'économie locale, et la protection et la consolidation du capital local : toutes choses qui visaient à résorber les déséquilibres internes, et à créer les conditions d'une adaptation continue de l'économie et de la société à la concurrence internationale.

À Taïwan, les dirigeants nationalistes, qui avaient en mémoire l'échec d'une étatisation trop forte de l'économie et d'une trop faible attention portée au développement rural en Chine, ont mis à profit l'aide des États-Unis pour enclencher un processus d'industrialisation fondé sur l'entreprise privée; mais ils n'ont pas séparé ce développement d'efforts considérables pour moderniser les infrastructures dans l'ensemble du pays et rentabiliser l'agriculture, en consolidant le système de petites exploitations sur lequel elle était fondée.

Et, alors que dans les années cinquante, ils avaient redressé l'économie sur une base autocentrée, quand le marché intérieur s'est trouvé saturé et que la possibilité d'une délocalisation des productions de main-d'œuvre s'est présentée, dans le cadre de la concurrence entre les États- Unis et le Japon, ils ont très vite cherché à tirer partie de la situation internationale, tout en poursuivant leur stratégie de développement par substitution d'importations. Ils ont alors encouragé les exportations et créé toutes les conditions nécessaires à l'attraction des firmes étrangères, par le biais de mesures incitatives et en engageant de gros travaux d'infrastructure - création de zones franches et de zones industrielles. Mais simultanément, ils ont développé une politique très dirigiste à l'égard des firmes étrangères, leur imposant des transferts de technologies et de compétences, et le recours à l'industrie locale pour une partie de leur production - le développement de la sous-traitance assurant à la fois la diversification de la production locale et son entrée dans la concurrence internationale. Ils ont également réparti les infrastructures industrielles dans tout le territoire, de façon à maximiser la position d'avantage comparé du pays en matière de coûts de main-d'œuvre, mettant à profit les traditions de la petite industrie en milieu rural, et 
assurant, dans le même temps, la résorption du sous-emploi et le maintien et l'élévation du niveau de vie dans les campagnes. Ils ont par ailleurs limité les possibilités d'implantation des firmes étrangères dans les branches de production où les firmes locales étaient déjà engagées, tout en protégeant fortement le marché intérieur.

Ceci n'est qu'un aspect de leur stratégie, mais qui a engagé le développement du pays tout entier.

À Singapour, les dirigeants gardaient en mémoire l'engagement révolutionnaire d'une large fraction de la population et restaient marqués par leurs origines familiales compradores ou administrateurs sous la colonisation britannique. Ils ont très rapidement engagé des investissements considérables pour attirer les firmes étrangères, afin que celles-ci fassent de la ville une de leurs bases de redéploiement industriel. Mais dans le même temps, ils ont effectué des travaux de rénovation urbaine et mené une politique de logements populaires de grande envergure assurant de meilleures conditions de vie à l'ensemble de la population, et permettant un contrôle social étroit. S'appuyant sur une très forte étatisation de l'économie, ils n'ont jamais séparé leur politique en faveur des firmes multinationales de politiques de valorisation des ressources humaines et d'un rapprochement des firmes locales et étrangères, dans le cadre d'entreprises à capitaux mixtes, pour maintenir la position d'avantage comparé de la ville-État au plan international.

On retrouve donc dans les stratégies de ces dirigeants deux mêmes constantes: la recherche de développement équilibré tant au plan économique, social que territorial ; l'aptitude à saisir toutes les opportunités offertes à différents niveaux et différentes échelles et à les valoriser en les " connectant » dans le sens de l'efficacité économique, mais aussi de l'efficacité sociale et politique. Gouvernements autoritaires, ceux-ci ont également su manier l'art du consensus.

Cette notion d'équilibre qui est au centre de leurs interventions, n'est sans doute pas sans liens avec la conception traditionnelle chinoise d'un État fort et vertueux, garant du bien-être social, que ces gouvernements ont largement repris pour légitimer leur pouvoir et assurer une paix sociale durable. Elle n'est pas très éloignée non plus de l'idée d'harmonie, au cœur du confucianisme, mais aussi de la médecine chinoise, qui pose comme condition au développement des capacités de l'être humain la maîtrise de ses forces intérieures et la protection des "dérèglements venus de l'extérieur " ${ }^{6}$.

31 Quant à l'art de combiner les "opportunités » offertes à différentes échelles - qui est aussi au centre des pratiques des entrepreneurs -, il a sans doute à voir avec la difficulté qu'ont les Chinois à penser le temps et l'espace comme deux entités abstraites et séparées. Dans la langue chinoise, le temps évoque «l'idée d'occasion, propice ou non pour une certaine action ", l'espace celle "de site favorable ou non, pour tel cas particulier ", et «le temps et l'espace ne sont jamais conçus indépendamment des actions concrètes qu'ils exercent (...) ni indépendamment des actions qu'on peut exercer sur eux $\gg .^{7}$ Et alors que dans la Chine ancienne, l'art de gouverner, le pouvoir, la réussite, relevaient de la capacité à " utiliser le destin en le tentant ", c'est-à-dire "à capter la force » des occasions que recèlent "les situations et conditions diverses de temps et de lieux " ${ }^{8}$, les entrepreneurs de Taïwan et de Singapour placent au premier plan de leur réussite la conjonction de trois éléments : " timing opportunity ", " geographical advantage », " harmonious human relationship ».

Cette pratique du temps et de l'espace, qui constitue un des éléments essentiels pour comprendre les fortes capacités de ces acteurs à s'adapter à la conjoncture et à assurer 
rapidement les reconversions que leur imposent les évolutions structurelles, renvoie néanmoins plus encore à la mémoire d'une diaspora, celle des Chinois d'outre-mer : mémoire d'une communauté marchande rejetée de Chine et qui a fondé son expansion sur des fonctions d'intermédiaire dans les échanges commerciaux au sein de l'Asie; mémoire d'une communauté souvent en position d'insécurité et d'exclusion politique dans les pays où elle était implantée, qui a réussi à s'imposer et à résister aux aléas d'une telle situation, en s'appuyant sur les réseaux de solidarité familiale et clanique et sur la dispersion de ses activités à l'échelle de l'Asie - voire du monde.

On retrouve là une façon de penser l'espace à vaste échelle et de jouer des opportunités extérieures, qui a été tout à fait décisive dans le modèle stratégique développé par les gouvernements et les entrepreneurs de Taïwan et de Singapour.

À Taïwan, le gouvernement nationaliste a fait très vite appel aux entrepreneurs chinois qui avaient fui la Chine communiste pour consolider sa base politique et mettre à profit leurs compétences industrielles, et il a constamment développé ses contacts avec la diaspora d'Asie - notamment de Hong-Kong - et des États-Unis pour soutenir le processus d'industrialisation engagé. Encore récemment, alors que l'industrie taïwanaise était en but à une perte d'avantage comparé de ses productions de maind'œuvre et confrontée à la nécessité de se reconvertir dans des secteurs de haute technologie, c'est en cherchant à attirer les Chinois formés aux États-Unis qu'il a tenté de stimuler les compétences locales dans ces secteurs.

À Singapour, le gouvernement a simultanément mis à profit la délocalisation des firmes étrangères sur son territoire et son capital relationnel en Asie pour renforcer, en les diversifiant, les fonctions régionales de la ville et son influence politique et économique vis-à-vis des pays environnants et plus particulièrement des Chinois de la diaspora.

C'est surtout du côté des entrepreneurs que la faculté d'établir des relations à différentes échelles et d'en jouer de façon variable selon les circonstances a été décisive. Elle est même une des clés essentielles pour comprendre l'ascension et la consolidation des groupes capitalistes de ces pays et leurs capacités d'affirmation économique.

37 À Taïwan, l'élite locale s'est trouvée initialement en but aux clivages et oppositions entre la population locale et les Chinois du continent soutenus par le pouvoir, et à une politique très discriminatoire du gouvernement nationaliste à son égard - celui-ci a apporté une aide directe très importante à une poignée d'entrepreneurs proches du pouvoir - : elle a cherché à s'imposer en mettant à profit son ancrage dans la société locale - notamment en milieu rural -, et en utilisant les réseaux commerciaux de la diaspora d'Asie pour exporter. Puis les entrepreneurs taïwanais ont mis en veilleuse pendant un temps ces réseaux, et se sont tournés vers les grandes sociétés commerciales américaines et japonaises pour entrer dans le processus d'exportation, tirant parti simultanément de leur ancrage dans la société et de leurs liens antérieurs avec les firmes nippones pour se placer comme sous-traitants des sociétés étrangères. Dans la période récente, qui exige des firmes locales de trouver de nouveaux marchés pour faire face aux protectionnismes, et de se reconvertir dans de nouvelles activités, les entrepreneurs ont fortement réactivé leurs relations avec la diaspora chinoise d'Asie et des États-Unis, mais aussi avec la Chine, pour délocaliser certaines de leurs productions dans des pays offrant des coûts de main-d'œuvre moins élevés, ou bénéficier de transferts de technologie et de parts de marchés; ils ont également cherché à tirer avantage des compétences des Chinois d'outre-mer dans de nouveaux 
secteurs. Mais cette internationalisation du capital - rendue possible par l'ouverture sur la diaspora- n'est pas dissociée d'une valorisation des compétences locales, permettant de renforcer les liens avec les firmes multinationales dans de nouveaux domaines. ${ }^{9}$

À Singapour, où la bourgeoisie était formée, de plus longue date, de Chinois d'outremer qui avaient fondé leur ascension sur le drainage des matières premières des pays environnants, et sur des fonctions de compradore en Asie, les entrepreneurs ont su tirer parti de leurs relations avec la communauté chinoise dans les pays voisins, et des politiques de développement engagées par les États pour étendre leur rôle dans les transactions entre ces pays et les firmes étrangères; ils ont dans le même temps maintenu une forte dispersion géographique de leurs activités, face à une forte étatisation de l'économie.

Les stratégies d'intégration à l'extérieur des classes dirigeantes et des entrepreneurs de Taïwan et de Singapour apparaissent ainsi totalement indissociables de leur mémoire, à des échelles temporelles et spatiales d'amplitude très variable. Et c'est certainement leur forte convergence et le fait qu'elles aient été en phase avec celles développées par les pays capitalistes centraux et les firmes transnationales qui peuvent permettre de comprendre leur « efficacité » dans la « réussite » de ces pays.

Géostratégies ou stratégies territoriales?

Si les stratégies étudiées concernent la façon dont les gouvernements et les entrepreneurs ont maîtrisé les relations entre leur espace national et le monde extérieur, peut-on pour autant les qualifier de stratégies territoriales?

Nous serions tentées de répondre positivement à cette question pour deux raisons : pour les gouvernements, elles ont été indissociables de leurs représentations territoriales et de modes d'interventions spécifiques d'organisation de l'espace interne ; pour les entrepreneurs, elles ont signifié des choix de localisation et la constitution de réseaux de relations au sein du territoire national ou de l'extérieur qui ont eu un impact important sur l'organisation et le rayonnement de ces espaces nationaux et qui contribuent à la formation des territoires transnationaux qui animent l'espace économique mondial.

\section{NOTES}

1. Cité par Jean LEVI, 1989. «Stratagèmes et prévoyance », in Les fonctionnaires divins, Paris, Seuil.

2. Op. cit.

3. Cf. PAIX Catherine et PETIT Michèle, 1987. « Un passé colonial et sa réinterprétation : la spécificité de la colonisation japonaise à Taïwan ». STRATES, 2, CNRS, Paris.

4. Pour reprendre une expression d'Hélène VERIN in Entrepreneurs, entreprises, histoire d'une idée, Paris, PUF.

5. Cf. CHESNEAUX Jean, 1982, Sun Yat-sen, Paris, Éditions Complexe.

6. ZAO WOU-KI et MARQUET Françoise, 1988. Zao Wou-Ki. Autoportrait, Paris, Fayard. 
7. GRANET Marcel, 1988. La pensée chinoise, Paris, Albin Michel.

8. Op. cit.

9. Cf. PAIX Catherine et PETIT Michèle, 1990. « Logiques héritées, nouvelles stratégies : les groupes capitalistes de Taïwan et de Singapour face aux défis de l'internationalisation », Paris. Tiers Monde, « Les bourgeoisies des Tiers Mondes d'hier à aujourd'hui », 124.

\section{RÉSUMÉS}

À Taïwan et à Singapour, gouvernements et entrepreneurs ont développé une stratégie d'intégration à l'économie et à l'espace mondial qui a consisté à retourner les rapports de domination/dépendance dans lesquels ils se sont trouvés placés, dans le sens d'un développement du territoire national. Les auteurs mettent en évidence les éléments qui sont entrés en jeu dans l'élaboration de ces stratégies. Celles-ci sont indissociables de la façon dont ces acteurs ont actualisé leur mémoire dans le contexte national et international qui a été le leur au moment de la constitution de ces États.

\section{Geostrategies of entreprises and governments in Taiwan et Singapore}

To integrate the world-wide space and economy, governments and entreprises in Taiwan and Singapore had developped specific strategies. Taking advantage of their foreign dependency, they have intended to act up balanced national developments as well as simultaneously national and international economical growths. Several factors which have comme into play and interfered in the elaboration of these strategies, are identified and argued by the authors.

\section{INDEX}

Keywords : taïwan, taiwan, Singapore, New Industrial Countries, State strategie, Firm strategy Mots-clés : Singapour, Nouveaux Pays Industriels, Stratégie gouvernementale, Stratégie de firme

\section{AUTEURS}

\section{CATHERINE PAIX}

Chargée de recherche au CNRS, elle travaille actuellement sur les stratégies des principaux acteurs du développement de plusieurs N.P.I. d'Asie - les entrepreneurs et la classe politique. Elle vient de diriger un ouvrage collectif sur Les bourgeoisies du Tiers Monde, à paraître au P.U.F.

\section{MICHÈLE PETIT}

Ingénieur au CNRS, elle poursuit actuellement des recherches sur la diaspora chinoise en Asie, en particulier sur la formation historique d'une couche d'entrepreneurs dans 
plusieurs N.P.I. Elle a collaboré à l'ouvrage collectif sur Les bourgeoisies du Tiers Monde, dirigé par C. PAIX. 